\title{
Adapt or Perish: Exercise referral schemes' operational changes during the Covid-19 pandemic.
}

Pre-print article published on: 02/10/2020 doi: https://doi.org/10.31236/osf.io/vnysx

Citation: Rowley, N., Shore, C., Buckley, B., Hesketh, K. (2020, October). Adapt or Perish: Exercise referral schemes' operational changes during the Covid-19 pandemic.

Co-author statement: CS initially presented the idea. NR led on the survey design, with CS, KH, and $\mathrm{BB}$ agreeing the final version. NR led writing up the short report, with $\mathrm{CS}, \mathrm{KH}$, and $\mathrm{BB}$ contributing and agreeing on the final version. All authors are aware of and agree to the submission to SportRxiv.

Twitter handles: @nikitarowley, @exe_therapy_col, @buckleybenjamin, @kathskth 


\section{BACKGROUND}

Covid-19 is having an extraordinary global impact, with many countries experiencing life-altering challenges due to the pandemic[1]. Three months post the initial outbreak in Wuhan, China, Covid-19 has become a global pandemic which has affected many countries across the world[1]. In late March 2020, Boris Johnson, the Prime Minister of United Kingdom (UK) announced a national lockdown, to attenuate the spread of the virus. This meant that leisure and sport facilities had to close their doors, having a likely negative impact on physical activity (PA) levels.

For many years, the world has been facing another pandemic; physical inactivity[2] and the increase of non-communicable diseases (NCDs)[3]. PA has been considered an effective prevention and management tool for a variety of NCDs[4]. It should be noted that having an NCD has been found to increase the risk of developing Covid-19[5]. Approximately, 20 million adults in the UK are physically inactive[6]. There is little knowledge on how this figure has been affected due to the Covid-19 pandemic, although preliminary Fitbit data has seen a decrease in PA globally[7].

\section{EXERCISE REFERRAL SCHEMES IN THE UK}

UK exercise referral schemes (ERSs) are delivered from community leisure and sport facilities, utilising PA and exercise to prevent, manage and treat NCDs. ERSs typically provide medically referred patients with the opportunity to take part in a structured, individualised exercise prescription in a safe and appropriate setting, such as leisure centre or gym. However, upon the closure of leisure facilities on $20^{\text {th }}$ March 2020, many schemes were faced with the option to adapt their delivery approaches, or face halting their scheme. 
Removing ERSs' capacity to deliver exercise prescriptions to at-risk patients in leisure and sport facilities, reduces the opportunity for patients to self-manage their NCDs and adhere to an exercise prescription. The pandemic has potentially had a huge impact on exercise prescription delivery which could have serious repercussions for patients' PA levels and health outcomes.

The authors conducted a service evaluation in May 2020 to review whether ERSs had adapted their delivery approaches and operations, allowing patients to continue participating within an exercise prescription. The information provided by schemes has been insightful and has provided some emphasis on how ERSs can be adaptable and offer flexibility- something that must be reviewed post-pandemic.

\section{HAVE EXERCISE REFERRAL SCHEMES ADAPTED THEIR DELIVERY APPROACHES DURING LOCKDOWN?}

A short survey was designed to evaluate delivery approaches used within UK-based ERSs during lockdown through a service evaluation; 28 schemes responded. It was found that $54 \%$ of schemes were still offering some level of service; with $46 \%$ of schemes not offering any service and halted operations. Of those schemes who were not able to operate (46\%), reasons for not being operational included lack of software capacity to deliver online sessions, and furloughed staff, not being able to offer any level of service. Of those schemes who were able to offer some service, adapted schemes so they could offer exercise videos for clients to follow, online virtual classes, leaflets, telephone support, support via updated websites, and in one site, deliver exercise equipment to patients to use at home. 
Additionally, there seems to be a shift from delivering a traditional ERSs to providing a wellbeing service, offering phone calls and emails to patients to help reduce social isolation. Large variability in the delivery approaches are seen, as such each ERS operates at a different capacity and operational standard. To date, these are the only known insights into ERS delivery during this pandemic.

\section{CHANGE TO DELIVERY APPORACHES}

Just over half of the schemes who responded have continued to provide some level of service during the lockdown period. With lockdown measures being lifted, leisure and sport facilities are working to make their environments safe for patients and clients to resume their exercise routines within their facilities. The information collected as part of the service evaluation has provided some useful insights of how schemes have adapted their provision during these challenging times. The flexible nature of the current ERS delivery could be desirable to future patients, who may not have the time or ability to attend ERS in person; some aspects of the current programme could therefore be maintained, or even preferred, post-lockdown. Moving forward, the authors are currently collecting and analysing more in-depth national data and a full research manuscript will be published later this year. Understanding the potential benefits of modifying ERSs during Covid-19 pandemic, may provide an opportunity for exercise referral stakeholders to revaluate their service delivery and increase flexibility in the way they operate in the future, postpandemic. 


\section{References}

[1] World Health Organization. Coronavirus disease (COVID-19) Pandemic.

2020. https://www.who.int/emergencies/diseases/novel-coronavirus-2019. Accessed $15 / 06 / 2020$.

[2] Ozemek C, Lavie CJ, Rognmo, O. Global physical activity levels - need for intervention. Prog Cardiovasc Dis 2020;62(2):102-107.

[3] Marmot, M, Bell, R. Social determinants and non-communicable diseases: time for integrated action. BMJ, 2019;364:1251.

[4] Pedersen, BK, Saltin, B. Exercise as medicine-evidence for prescribing exercise as therapy in 26 different chronic diseases. Scand J Med Sci Sports 2015:25(3):1-72.

[5]Basu, S. Non-communicable disease management in vulnerable patients during Covid19. Indian Journal of Medical Ethics 2020:5(2).

[6] ukactive. Moving Communities: Active Leisure Trends 2018 Report. 2020, London: ukactive.

[7] Fitbit. The impact of Coronavirus on global activity. 2020. https://blog.fitbit.com/covid19-global-activity/. Accessed June 16, 2020. 
Contributor information: CS initially presented the idea. NR led on the survey design, with $\mathrm{CS}, \mathrm{KH}$, and $\mathrm{BB}$ agreeing the final version. NR led writing up the short report, with $\mathrm{CS}, \mathrm{KH}$, and BB contributing and agreeing on the final version.

Funding information: $n / a$

Conflicts of interest- no conflicts of interest between authors.

Availability of data - Data has been made public https://osf.io/ausb4/?view only=0c50c0c059cc4262a01d15b052a22519

Ethical approval information- Formal ethical approval was not required for this short report. This piece of work did not need to undergo a formal committee review and is in essence a priori considered 'approved' ethically. 